\title{
MARKOV PARTITIONS FOR THE TWO-DIMENSIONAL TORUS
}

\author{
MARK R. SNAVELY
}

(Communicated by Kenneth R. Meyer)

\begin{abstract}
We examine Markov partitions for hyperbolic automorphisms of $\mathbb{T}^{2}$ in the spirit of Adler, Weiss, and others and give necessary conditions on the transition matrix of a Markov partition for a given automorphism. We give necessary and sufficient conditions for partitions with two connected rectangles.
\end{abstract}

\section{BACKGROUND DEFINITIONS}

We begin by briefly reviewing the notions of a hyperbolic automorphism of $\mathbb{T}^{2}\left(\mathbb{T}^{2}=\mathbb{R}^{2} / \mathbb{Z}^{2}\right.$ where $\left.\mathbb{R}^{2} \stackrel{\pi}{\rightarrow} \mathbb{R}^{2} / \mathbb{Z}^{2}\right)$ and that of a Markov partition. For a more detailed introduction see [AW, D, Sn]. Let $\mathscr{A}$ be a $2 \times 2$ matrix with integer entries. Suppose also that $\operatorname{det}(\mathscr{A})= \pm 1$ so that $\mathscr{A}^{-1}$ is an integer matrix. In other words, $\mathscr{A} \in \mathrm{GL}(2, \mathbb{Z})$. We further suppose that none of the eigenvalues of $\mathscr{A}$ have modulus 1 . Therefore by the Perron-Froebenius Theorem we have real eigenvalues $\lambda_{u}$ and $\lambda_{s}$ satisfying $\left|\lambda_{u}\right|>1>\left|\lambda_{s}\right|>0$. We call $\lambda_{u}$ the unstable eigenvalue of $\mathscr{A}$ and $\lambda_{s}$ the stable eigenvalue of $\mathscr{A}$. The corresponding eigenvectors, $\vec{v}_{u}$ and $\vec{v}_{s}$, are called the unstable and stable eigenvectors respectively. We note at this time that $\operatorname{Tr}(\mathscr{A}) \neq 0$. If $\operatorname{Tr}(\mathscr{A})>0$, then $\lambda_{u}>0$ and if $\operatorname{Tr}(\mathscr{A})<0$, then $\lambda_{u}<0 . \mathscr{A}$ induces an automorphism of $\mathbb{T}^{2}$, which we will also denote $\mathscr{A}$. We define $W^{u}(x)$ for $x \in \mathbb{T}^{2}$ as the projection of a line through $\pi^{-1} x$ parallel to $\vec{v}_{u}$ with $W^{S}(x)$ defined similarly.

Definition 1.1. We define $\mathscr{P}=\left\{R_{i}\right\}_{i=1}^{n}$ to be a Markov partition for $\mathscr{A}$ if the following are true:

(i) $\mathbb{T}^{2}=\bigcup_{i=1}^{n} R_{i}$;

(ii) $R_{i}=\overline{\text { interior }\left(R_{i}\right)}$;

(iii) interior $\left(R_{i}\right) \cap$ interior $\left(R_{j}\right)=\varnothing, i \neq j$;

(iv) if $x, y \in R_{i}$, then $W^{u}\left(x, R_{i}\right) \cap W^{s}\left(y, R_{i}\right)=z \in R_{i}$ (the intersection is a single point $z)$;

(v) $\mathscr{A}\left(W^{u}\left(x, R_{i}\right)\right) \supset W^{u}\left(\mathscr{A} x, R_{j}\right)$ where $x \in R_{i}$ and $\mathscr{A} x \in R_{j}$;

Received by the editors December 5, 1989; the contents of this paper were presented at the Conference/Workshop on Ergodic Theory and Symbolic Dynamics, University of Washington, Seattle, on June 21,1989 . This conference was sponsored in part by the NSF.

1980 Mathematics Subject Classification (1985 Revision). Primary 58F15. 
(vi) $\mathscr{A}\left(W^{s}\left(x, R_{i}\right)\right) \subset W^{s}\left(\mathscr{A} x, R_{j}\right)$ where $x \in R_{i}$ and $\mathscr{A} x \in R_{j}$; where $W^{u}\left(x, R_{i}\right)=W_{\varepsilon}^{u}(x) \cap R_{i}$.

The $R_{i}$ 's defined above are called rectangles and for automorphisms of $\mathbb{T}^{2}$ they can be unions of actual rectangles in the geometric sense. This is true in this paper, and we assume that all rectangles are connected. Disconnected rectangles are covered in [Sn]. Connected rectangles have two sides parallel to a line through $\mathbf{0}$ in the direction of $\vec{v}_{u}$ and two sides parallel to a line through 0 in the direction of $\vec{v}_{s}$. The boundary of $\mathscr{P}$, which is the union of the boundaries of the $R_{i}$ 's, can then be written as $\partial \mathscr{P}=\partial_{u} \mathscr{P} \cup \partial_{s} \mathscr{P}$ where $\partial_{u} \mathscr{P}$ is the union of the unstable sides of the $R_{i}$ and $\partial_{s} \mathscr{P}$ defined similarly. From the definition of Markov partition, we see that (i) $\mathscr{A}^{-1}\left(\partial_{u} \mathscr{P}\right) \subset \partial_{u} \mathscr{P}$ and (ii) $\mathscr{A}\left(\partial_{s} \mathscr{P}\right) \subset \partial_{s} \mathscr{P}$. This allows us to define the following:

Definition 1.2. The unstable core of $\mathscr{P}$, denoted $\mathscr{U}$, is defined as $\mathscr{U}=$ $\bigcap_{i=1}^{\infty} \mathscr{A}^{-i}\left(\partial_{u} \mathscr{P}\right)$.

Similarly, define the stable core of $\mathscr{P}$, denoted $\mathscr{S}$, as $\mathscr{S}=\bigcap_{i=1}^{\infty} \mathscr{A}^{i}\left(\partial_{s} \mathscr{P}\right)$. We then define the core of $\mathscr{P}$, denoted $\mathscr{C}$, as $\mathscr{C}=\mathscr{U} \cup \mathscr{S}$.

From (v) and (vi) in the definition of Markov partition, we know that if $\mathscr{A}\left(R_{i}\right)$ intersects $R_{j}$, then $\mathscr{A}\left(R_{i}\right)$ crosses $R_{j}$ from one end to the other in the unstable direction. Hence, we can define a transition matrix as follows:

Definition 1.3. We define the Markov matrix of a Markov partition $\mathscr{P}$ with $n$ rectangles for $\mathscr{A}$ by $m_{i, j}=\left\{\right.$ the number of times $\mathscr{A}\left(R_{i}\right)$ crosses the interior of $\left.R_{j}\right\}$ for $1 \leq i, j \leq n$.

Example 1.4. Let $\mathscr{A}=\left[\begin{array}{ll}2 & 1 \\ 1 & 1\end{array}\right]$. The eigenvalues of $\mathscr{A}$ are $\lambda_{u}=(3+\sqrt{5}) / 2$ and $\lambda_{s}=(3-\sqrt{5}) / 2$ with corresponding eigenvectors $[(\sqrt{5}-1) / 2]$ and $[(-\sqrt{5}-1) / 2]$ respectively.

Figure 1.5 is a Markov partition $\mathscr{P}$ for $\mathscr{A}$ and $\mathscr{A}(\mathscr{P})$. We view $\mathbb{T}^{2}$ as $[0,1] \times[0,1]$ with opposite sides identified.

We see that $\mathscr{A}\left(R_{1}\right)$ crosses $R_{1}$ twice and $R_{2}$ once and $\mathscr{A}\left(R_{2}\right)$ crosses $R_{1}$ and $R_{2}$ each once giving us $M=\left[\begin{array}{ll}2 & 1 \\ 1 & 1\end{array}\right]$. Notice that if we reverse the labeling of $R_{1}$ and $R_{2}$ then $M=\left[\begin{array}{ll}1 & 1 \\ 1 & 2\end{array}\right]$.
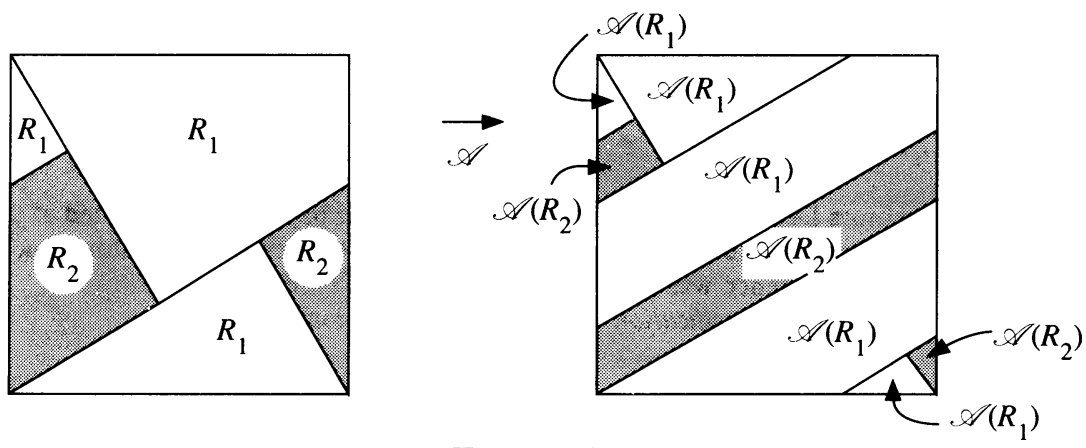

FIGURE 1.5 


\section{The eigenvalues of The Markov matrix}

The goal of this paper is to say what we can about the Markov matrix $M$. The following theorem tells us about the eigenvalues of $M$. First, we need the following lemma due to Matt Stafford.

Lemma 2.1 [St]. Let $h: B \rightarrow B, B$ finite. Then every eigenvalue of

$$
h_{*}: \widetilde{H}_{0}(B) \rightarrow \widetilde{H}_{0}(B)
$$

is either 0 or a root of unity. The multiplicity of the 0 eigenvalue is equal to the number of preperiodic points in $B$. The multiplicity of the eigenvalue 1 is equal to (\# of periodic orbits in $B$ ) -1 .

Proof. If $B$ is a finite union of periodic orbits, $\left.h\right|_{B}$ can be represented by a permutation matrix. (Think of each point in $B$ as a basis element.) Clearly, this permutation is cyclic iff $B$ consists of a single periodic orbit.

If $B$ contains preperiodic points as well, then $\left.h\right|_{B}$ can be represented by a matrix of the form

$$
Q=\left[\begin{array}{cccc}
P & 0 & 0 & 0 \\
* & 0 & 0 & 0 \\
* & * & \ddots & 0 \\
* & * & * & 0
\end{array}\right]
$$

where $P$ is a permutation matrix representing the action of $h$ on the periodic points in $B$. The rows below $P$ represent the action of the preperiodic points in $B$. (Here, one must be careful about the ordering of the basis elements: if $x$ and $y$ are preperiodic and $h(x)=y, y$ should be listed before $x$.) It is clear from the block form of $Q$ that $\chi_{Q}(t)=\chi_{P}(t)(-t)^{n}$, where $n$ is the number of preperiodic points in $B$. Note that the multiplicity of the root 1 in $\chi_{P}(t)$ and thus in $\chi_{Q}(t)$ is equal to the number of periodic orbits in $B$.

Let $b_{0}, b_{1}, \ldots, b_{p-1}$ be the elements of $B . h$ induces endomorphisms of $H_{0}(B) \cong \mathbb{Z}^{p}$ and $\widetilde{H}_{0}(B) \cong \mathbb{Z}^{p-1}$. With respect to the basis $b_{0}, b_{1}, \ldots, b_{p-1}$, the former is represented by the matrix $Q$ described above. Let $Q^{\prime}$ represent the same transformation in the basis $\sum_{i=0}^{p-1} b_{i}, b_{1}-b_{0}, b_{2}-b_{0}, \ldots, b_{p-1}-b_{0}$. $Q^{\prime}$ is of the form

$$
Q^{\prime}=\left[\begin{array}{ll}
1 & * \\
0 & F
\end{array}\right]
$$

To prove this, it suffices to show that $V=\operatorname{span}\left\{b_{1}-b_{0}, b_{2}-b_{0}, \ldots, b_{p-1}-b_{0}\right\}$ is an invariant subspace under $h_{*} . h_{*}\left(b_{i}-b_{0}\right)=h\left(b_{i}\right)-h\left(b_{0}\right)$. If $h\left(b_{i}\right)=h\left(b_{0}\right)$, this difference is $0 \in V$. Otherwise, $h_{*}\left(b_{i}-b_{0}\right)$ is in the set $\left(b_{i}-b_{j} \mid i \neq j\right)$, which is clearly contained in $V$. Thus $h_{*}$ maps the basis elements of $V$ into $V$; it follows that $h_{*}(V) \subset V$.

The reason for this change of basis can now be made clear: $b_{1}-b_{0}, b_{2}-$ $b_{0}, \ldots, b_{p-1}-b_{0}$ form a basis of $\widetilde{H}_{0}(B)$ and $h_{*}: \widetilde{H}_{0}(B) \rightarrow \widetilde{H}_{0}(B)$ is represented by the submatrix $F$ (of $Q^{\prime}$ above) in this basis. Further, $\chi_{F}(t)=$ $\chi_{Q^{\prime}}(t) /(1-t)=\chi_{Q}(t) /(1-t)$. Thus every eigenvalue of $F$ must be 0 or a root 
of unity. The multiplicity of the eigenvalue 0 for the $p \times p$ matrix $Q$ is not decreased when passing to the $(p-1) \times(p-1)$ matrix $F$. But the multiplicity of the eigenvalue 1 is one less for $F$ than for $Q$. This verifies the claim about the multiplicities of the eigenvalues 0 and 1 and completes the proof of the lemma.

We use both this lemma and its proof in the following theorem:

Theorem 2.2. Let $\mathscr{P}=\left\{R_{i}\right\}_{i=1}^{n}$ be a Markov partition for $\mathscr{A}$ where $\mathscr{A}$ is a hyperbolic automorphism of $\mathbb{T}^{2}$. If $\operatorname{Tr}(\mathscr{A})>0$, then the eigenvalues of $M$ are $\lambda_{u}, \lambda_{s}$, together with 0 's and roots of unity. If $\operatorname{Tr}(\mathscr{A})<0$, then the eigenvalues of $M$ are $-\lambda_{u},-\lambda_{s}$, together with 0 's and roots of unity.

Proof. A detailed proof of this theorem can be found in [Sn]. We give the proof under the further assumption that both $\partial_{u} \mathscr{P}$ and $\partial_{s} \mathscr{P}$ are connected.

Suppose $\mathscr{P}$ has $n$ rectangles, and that $\operatorname{Tr}(\mathscr{A})>0$. Let $X=\mathbb{T}^{2}-\partial_{u}^{\circ} \mathscr{P}$, and $A=X \cap \partial_{s}^{\circ} \mathscr{P}$. By $\partial_{u}^{\circ} \mathscr{P}$ we mean consider $\partial_{u} \mathscr{P}$ as a line segment and $\partial_{u}^{\circ} \mathscr{P}$ is then the interior of that line segment. In our case, this is just $\partial_{u} \mathscr{P}$ without the endpoints. $A$ has $n-1$ components and therefore, is homologous to $n-1$ points. The best way to see that $A$ has $n-1$ components is to visualize yourself walking the length of $\partial_{s} \mathscr{P}$ looking say to the left, counting rectangles once you have crossed them. You pass each rectangle once. Each rectangle is counted at a point where $\partial_{u} \mathscr{P}$ crosses $\partial_{s} \mathscr{P}$ completely except two; one is counted at a point where $\partial_{u} \mathscr{P}$ has an endpoint in $\partial_{s} \mathscr{P}$ and the last one sees an endpoint of $\partial_{s} \mathscr{P}$ in $\partial_{u} \mathscr{P}$. Now $n=$ \{number of crossings $\}+2$, hence number of crossings $=n-2$. Also, the number of components of $A$ is number of crossings +1 . Therefore, the number of components in $A$ is $n-1 . X$ is homologous to $\mathbb{T}^{2}-$ one point $\}$. Then we know that $\widetilde{H}_{1}(A)=0$, $\widetilde{H}_{1}(X)=\mathbb{Z}^{2}, \widetilde{H}_{0}(A)=\mathbb{Z}^{n-2}$, and $\widetilde{H}_{0}(X)=0$. The exact relative homology sequence for a pair $(X, A)$ gives us the following commutative diagram.

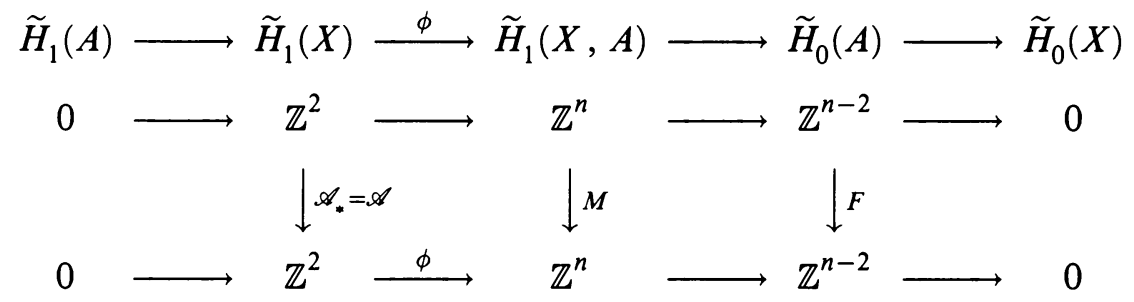

Because $\mathbb{Z}^{m}$ is free abelian for $m \geq 0$, the top line of the above sequence is split exact and hence $\widetilde{H}_{1}(X, A)=\mathbb{Z}^{n} . \mathscr{A}_{*}$ is the map induced on $\widetilde{H}_{1}(X)$ by $\mathscr{A}$, which is in fact $\mathscr{A}$ itself. One set of generators for $\widetilde{H}_{1}(X, A)$ is a line segment across each rectangle in the unstable direction so the induced map on homology is just $M . F$ is from Lemma 2.1. Because the top line of the above sequence is split exact, with possibly a different choice of basis, $M$ can 
be written in the form:

$$
\left[\begin{array}{ll}
\mathscr{A} & 0 \\
* & F
\end{array}\right]
$$

Thus the eigenvalues of $M$ are exactly the eigenvalues of $\mathscr{A}\left(\lambda_{u}\right.$ and $\left.\lambda_{s}\right)$ together with the eigenvalues of $F$ (roots of unity and zeros) when $\operatorname{Tr}(\mathscr{A})>0$. When $\operatorname{Tr}(\mathscr{A})<0$, the induced map on $\widetilde{H}_{1}(X, A)$ is $-M$ (because $\lambda_{u}<0$, $\mathscr{A}$ reverses the orientation of the generators in $\left.\widetilde{H}_{1}(X, A)\right)$ so in the diagram replace $M$ with $-M$ and the theorem follows similarly, thus concluding the proof for our case; namely, $\partial_{u} \mathscr{P}$ and $\partial_{s} \mathscr{P}$ being connected.

The proof of this theorem gives us the following two results:

Corollary 2.3. Let $\mathscr{P}$ be a Markov partition for $\mathscr{A}$ with $n>2$ rectangles. Then the Markov matrix $M$ is similar over $\mathbb{Z}$ to an $n \times n$ matrix of the following form:

$$
\left[\begin{array}{cccc}
\mathscr{A} & 0 & 0 & 0 \\
* & A_{1} & 0 & 0 \\
* & * & \ddots & 0 \\
* & * & * & A_{k}
\end{array}\right]
$$

where the eigenvalues of the nonzero $A_{i}$ are either $\{$ all the pth roots of unity for some $p\}$ or $\{$ all the pth roots of unity except 1 for some $p\}$.

Proof. By noting that $F$ in the above proof has as eigenvalues only roots of unity and zeros, the corollary follows directly from the proof of Theorem 2.2 and linear algebra [N].

Roy Adler, in an as yet unpublished work, proved that every toral automorphism $\mathscr{A}$ has a Markov partition with two rectangles. He in fact proved the existence of a Markov partition for which the Markov matrix is $\mathscr{A}$ itself. We use the first result to give us the following corollary, which is Corollary 2.3 in the $2 \times 2$ case:

Corollary 2.4. Let $\mathscr{P}$ be a Markov partition for $\mathscr{A}$ with two rectangles and Markov matrix $M$. Then if $\operatorname{Tr}(\mathscr{A})>0$, there exists $\phi \in \operatorname{GL}(2, \mathbb{Z})$ such that $\phi^{-1} \mathscr{A} \phi=M$. Similarly, if $\operatorname{Tr}(\mathscr{A})<0$, there exists $\phi \in \mathrm{GL}(2, \mathbb{Z})$ such that $\phi^{-1} \mathscr{A} \phi=-M$.

Proof. Letting $X$ and $A$ be as in Theorem 2.2, with $n=2$ and $\operatorname{Tr}(\mathscr{A})>0$, we have the following diagram.

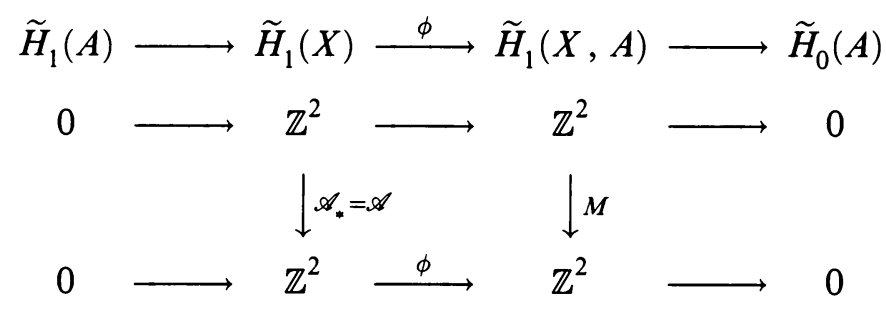


$\phi$ is an isomorphism hence in $\operatorname{GL}(2, \mathbb{Z})$ and in the corollary is proven for $\operatorname{Tr}(\mathscr{A})>0$ with the proof for $\operatorname{Tr}(\mathscr{A})<0$ being entirely similar.

At this time we note that $\partial_{u} \mathscr{P}$ and $\partial_{s} \mathscr{P}$ being connected implies that $\mathscr{C}(\mathscr{P})$ is at least one fixed point and at most two fixed points. Also, the maps $\left.\mathscr{A}\right|_{\mathscr{U}}$ and $\left.\mathscr{A}\right|_{S}$ together with the signs of $\lambda_{u}$ and $\lambda_{s}$ determine the nonzero eigenvalues of $M$. This relationship is explored in [Sn].

\section{Some ALgebraic Results}

The natural question to ask at this point is whether or not the converse of either of these corollaries is true. Let us examine the $2 \times 2$ case. In order to do so we need the following algebraic results.

3.1. Let $\mathscr{A} \geq 0$ be an element of $\mathrm{GL}(2, \mathbb{Z})$ and suppose $\mathscr{A}$ is hyperbolic. Then $\mathscr{A}$ has a dominant row and a dominant column; i.e. the first column of $\mathscr{A}=\left[\begin{array}{ll}a & b \\ c & d\end{array}\right]$ is dominant if $a \geq b$ and $c \geq d$ and similarly for a row being dominant.

Proof. Suppose $\mathscr{A}$ does not have a dominant column. Then either (i) $a>b$ and $c<d$ or (ii) $b>a$ and $d<c$.

Case (i). $a>b$ and $c<d \Rightarrow a \geq b+1 \Rightarrow a d>b c+c \Rightarrow a d-b c>c$. Therefore, since $\operatorname{det}(\mathscr{A})= \pm 1$ and $c \geq 0$, we have that $c=0 \Rightarrow a=d=1 \Rightarrow$ $b=0$ (since $a>b) \Rightarrow \mathscr{A}=\left[\begin{array}{ll}1 & 0 \\ 0 & 1\end{array}\right]$, which is a contradiction to the hyperbolicity of $\mathscr{A}$.

Case (ii). $b>a$ and $d<c \Rightarrow b \geq a+1 \Rightarrow b c>a d+d \Rightarrow b c-a d>d$. Therefore, since $\operatorname{det}(\mathscr{A})= \pm 1$ and $d \geq 0$, we have that $d=0 \Rightarrow b=c=1 \Rightarrow$ $a=0$ (since $b>a) \Rightarrow \mathscr{A}=\left[\begin{array}{ll}0 & 1 \\ 1 & 0\end{array}\right]$, which is a contradiction.

3.2. Suppose $\mathscr{A} \in \mathrm{GL}(2, \mathbb{Z}), \mathscr{A} \geq 0, \mathscr{A} \neq\left[\begin{array}{ll}1 & 0 \\ 0 & 1\end{array}\right]$. Then $\mathscr{A}=\prod_{i=1}^{n}\left[\begin{array}{c}x_{i} \\ 1 \\ 1\end{array}\right]$ where $x_{i}=0$ or 1 for $1 \leq i \leq n$.

Sketch of proof. We can see the above by observing the following:

$$
\left[\begin{array}{ll}
a & b \\
c & d
\end{array}\right]=\left[\begin{array}{ll}
x & 1 \\
1 & 0
\end{array}\right]\left[\begin{array}{ll}
c & d \\
y & z
\end{array}\right]
$$

where if $a \geq c$ and $b \geq d$ then let $x=1, y=a-c$, and $z=b-d$ thus reducing the entries in the dominant row. Otherwise let $x=0, y=a$, and $z=b$. We can then view this factorization as a sequence of 1's and 0 's $\left\{x_{i}\right\}_{i=1}^{n}$ and this factorization is unique up to two consecutive 0 's $\left(\left[\begin{array}{ll}0 & 1 \\ 1 & 0\end{array}\right]^{2}=\left[\begin{array}{ll}1 & 0 \\ 0 & 1\end{array}\right]\right)$. We call $\left\{x_{i}\right\}_{i=1}^{n}$ the defining sequence for $\mathscr{A}$. From here on we denote $\left[\begin{array}{ll}0 & 1 \\ 1 & 0\end{array}\right]$ as $x,\left[\begin{array}{ll}1 & 1 \\ 1 & 0\end{array}\right]$ as $y$, and $\left[\begin{array}{ll}0 & 1 \\ 1 & 1\end{array}\right]$ as $y^{\prime}$.

3.3. Under the same hypotheses as in 3.2 , we see that $\mathscr{A}$ also factors as follows: We define a shear matrix to be a matrix with ones on the diagonal and a single one off the diagonal while all other off-diagonal entries are 0 . Note that if a shear matrix has a one as its $i$, jth entry then its inverse has a minus one as its 
$i$, jth entry. If $\mathscr{A} \neq x$, then $\mathscr{A}=\prod_{i=1}^{m} M_{i}$ where $M_{i}$ is a $2 \times 2$ shear matrix for $1 \leq i \leq m-1$ and $M_{m}$ is either a $2 \times 2$ shear matrix or $y$ or $y^{\prime}$.

Proof. We can see that $x y=y^{\prime} x, y x=\left[\begin{array}{ll}1 & 1 \\ 0 & 1\end{array}\right]$, a shear, and $y^{\prime} x=\left[\begin{array}{ll}1 & 0 \\ 1 & 1\end{array}\right]$, a shear. Given the defining sequence for $\mathscr{A},\left\{x_{i}\right\}_{i=1}^{n}$, we replace 1's with $y$ 's and 0 's with the $x$ 's. Now using the first relation above, put the sequence in the form $s_{1} x s_{2} x \cdots s_{k} x s_{k+1} s_{k+2} \cdots s_{m}$ where $s_{i}$ can be either $y$ or $y^{\prime}$ for $1 \leq i \leq m$. (If there are no $x$ 's in the sequence, we let $k=0$.) If $m=k$ or $m=k+1$ then we are done. If $m>k+1$ then insert $x x$ between $s_{k+2 j-1}$ and $s_{k+2 j}$ for $1 \leq j \leq\lfloor m-k / 2\rfloor$ (where $\lfloor r\rfloor=\{$ the largest integer smaller than $r\}$ ), replace $x s_{k+2 j}$ with $s x$ where $s$ is either $y$ or $y^{\prime}$ and we are done.

3.4. Suppose $\mathscr{A}$ and $\mathscr{B}$ are nonnegative, hyperbolic matrices in $\mathrm{GL}(2, \mathbb{Z})$. The following are equivalent:

(i) $\mathscr{A}$ and $\mathscr{B}$ are similar over $\mathbb{Z}$.

(ii) There is a matrix $P \geq 0, P \in \mathrm{GL}(2, \mathbb{Z})$, such that $P^{-1} \mathscr{A} P=\mathscr{B}$.

(iii) The defining sequence for $\mathscr{A}$ is a cyclic permutation of the defining sequence for $\mathscr{B}$ up to two consecutive 0 's.

Sketch of proof. (ii) $\Leftrightarrow$ (i) is obvious as (iii) $\Leftrightarrow$ (ii). We then must show that (i) $\Rightarrow$ (iii). One way to do this is to see that (i) $\Rightarrow$ shift equivalent over $\mathbb{Z} \Rightarrow[K R]$ shift equivalent over $\mathbb{Z}^{+} \Rightarrow$ (iii). It can also be proven directly by letting $w=\left[\begin{array}{cc}-1 & 0 \\ 0 & 1\end{array}\right]$ and showing that the group generated by $x, y$, and $w$ has a solvable word problem and is in fact $\operatorname{GL}(2, \mathbb{Z})$. Then using this machinery and a defining sequence for any element of $\operatorname{GL}(2, \mathbb{Z})$ involving $x, y$, and $w$, show (i) $\Leftrightarrow$ new defining sequence is cyclic permutation $\Leftrightarrow$ (iii).

\section{Partitions with two Rectangles}

We are now ready to begin examining partitions with two rectangles with the following lemma.

Lemma 4.1. Let $\mathscr{P}$ be a Markov partition with two rectangles for $\mathscr{A}$ with Markov matrix $M$ and suppose there is a shear matrix $S$ and a matrix $M^{\prime} \geq 0$ such that $S^{-1} M S=M^{\prime}$. Then there is a Markov partition $\mathscr{P}^{\prime}$ for $\mathscr{A}$ with Markov matrix $M^{\prime}$.

Proof. We give the proof for $S=\left[\begin{array}{ll}1 & 1 \\ 0 & 1\end{array}\right]$ and $\operatorname{Tr}(\mathscr{A})>0$ with the proofs for $\left[\begin{array}{ll}1 & 0 \\ 1 & 1\end{array}\right]$ and $\operatorname{Tr}(\mathscr{A})<0$ being similar. Suppose first that $\mathscr{C}(\mathscr{P})=$ origin . Consider

$$
S^{-1} M S=\left[\begin{array}{cc}
1 & -1 \\
0 & 1
\end{array}\right]\left[\begin{array}{ll}
a & b \\
c & d
\end{array}\right]\left[\begin{array}{ll}
1 & 1 \\
0 & 1
\end{array}\right]=\left[\begin{array}{cc}
a-c & a-c+b-d \\
c & c+d
\end{array}\right]=M^{\prime} .
$$

In order for this matrix to be nonnegative, row 1 must be the dominant row in $M$. Row 1 being dominant means that $\mathscr{A}\left(R_{1}\right)$ crosses each rectangle at least as many times as $\mathscr{A}\left(R_{2}\right)$, which says that $R_{1}$ is strictly longer than $R_{2}$ in the unstable direction. If $R_{1}$ is strictly longer than $R_{2}$, we can divide $R_{1}$ into 
two pieces, $R_{1}^{\prime}$ and $L$ as shown in Figure 4.2b. To divide $R_{1}$ in this manner, draw $R_{1}$ and $R_{2}$ with their unstable sides furthest from the origin side by side (Figure 4.2a). We then extend segment $\overline{C B}$ to $\overline{C D}$ and now have a Markov partition $\mathscr{T}$ with three rectangles, $R_{1}^{\prime}, L$, and $R_{2}$. The Markov matrix for $\mathscr{T}$ is

$$
M(\mathscr{T})=\left[\begin{array}{ccc}
a-c & a-c & b-d \\
c & c & d \\
c & c & d
\end{array}\right] .
$$

For row $3, \mathscr{A}\left(R_{2}\right)$ crosses $R_{2}$ the same number of times as in $\mathscr{P}$, and $L$ and $R_{1}^{\prime}$ the number of times it crossed $R_{1}$ in $\mathscr{P}$. For row $2, \mathscr{A}(L)$ crosses the same rectangles as $\mathscr{A}\left(R_{2}\right)$; because of the expansive nature of the unstable boundary, the boundary between $R_{2}$ and $L$ (segment $\overline{A B}$ ) gets mapped to the interior of any rectangle it crosses (see Example 1.4). $\mathscr{A}\left(R_{1}^{\prime}\right)$ then crosses everything that $R_{1}$ used to cross that is not crossed by $\mathscr{A}(L)$. Then we have seen that $S^{-1} M S$ being nonnegative (in particular, multiplication on the left by $S^{-1}$ ) means that we can split $R_{1}$ into two rectangles, one of which has the same "personality" as $R_{2}$. Now we can remove segment $\overline{A B}$ from $\mathscr{T}$ and get a new partition $\mathscr{P}^{\prime}$ (Figure 4.2c). This is possible because $\mathscr{A}(\overline{A B}) \cap \partial_{u} \mathscr{P}=\varnothing$. We claim that the Markov matrix for $\mathscr{P}^{\prime}$ is $M^{\prime}$. The rectangles for $\mathscr{P}^{\prime}$ are $R_{2}^{\prime}=R_{2} \cup L$ and $R_{1}^{\prime} . R_{2}^{\prime}$ is crossed by everything that crossed either $R_{2}$ or $L$, hence, column 2 of $M^{\prime}$ is $\{$ column 2 of $M(\mathscr{T})\}+\{$ column 3 of $M(\mathscr{T})\}$ with either the second or third entry deleted\}. Column 1 of $M^{\prime}$ is \{column 1 of $M(\mathscr{T})$ with either the second or third entry deleted\}. Hence,

$$
M^{\prime}=\left[\begin{array}{cc}
a-c & a-c+b-d \\
c & d+c
\end{array}\right] .
$$

This concludes the proof for $\mathscr{C}(\mathscr{P})=$ origin $\}$. If $\mathscr{C}=$ any fixed point not the origin $\}$, then by translation the above proof works. Then we must deal with the case when $\mathscr{C}(\mathscr{P})=\{$ two fixed points $\}$. Notice that if there are more than two fixed points in the core or any periodic point of higher period, then there are more than two rectangles [Sn]. We assume that one of these fixed points is the origin. The only part of the above proof that we must modify is how to
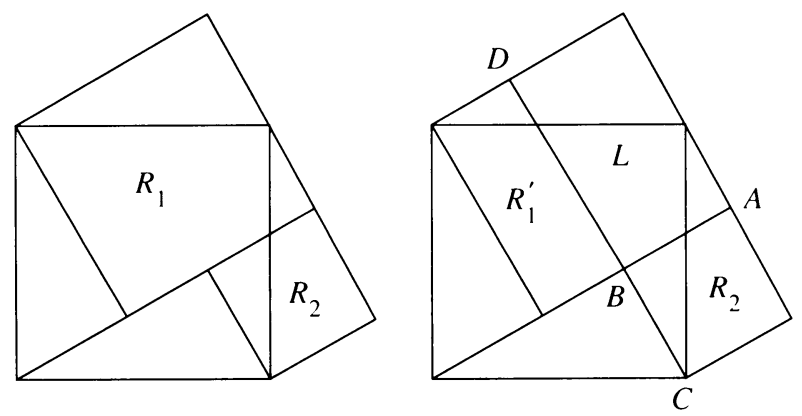

FIGURES $4.2 \mathrm{a}$ AND $4.2 \mathrm{~b}$ 


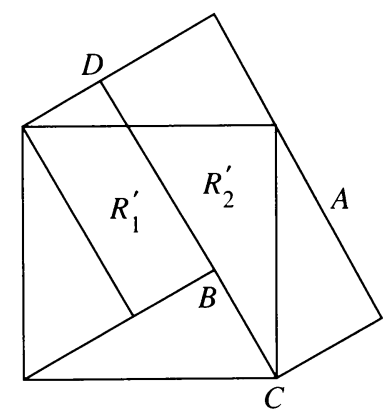

FIGURE 4.2c

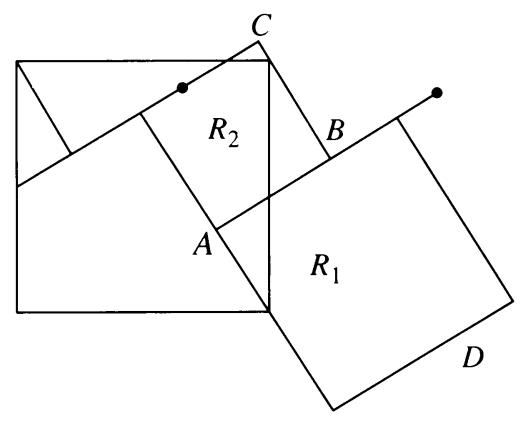

FIGURE 4.3

divide $R_{1}$. First, we must have $\mathscr{U}=$ \{one fixed point $\}$ and $\mathscr{S}=\{$ the other fixed point $\}$. We assume WLOG that $\mathscr{S}=0$ and $\mathscr{U}=\{$ the other fixed point $\}$, call it $q$. We want to draw $\mathscr{P}$ so that there is no fixed point on $\overline{A B}$ as in Figure 4.3. It is then possible to extend $\overline{C B}$ to $\overline{C D}$ and obtain a partition $\mathscr{T}$. then remove $\overline{A B}$ as above.

We use the lemma to prove the following proposition that in turn gives us the theorem.

Proposition 4.4. Let $\mathscr{P}$ be a Markov partition with two rectangles for $\mathscr{A}$ with Markov matrix $M$ and suppose there is a matrix $M^{\prime} \geq 0$ and $\phi \in \mathrm{GL}(2, \mathbb{Z})$ such that $\phi^{-1} M \phi=M^{\prime}$. Then there exists a Markov partition $\mathscr{P}^{\prime}$ for $\mathscr{A}$ with Markov matrix $M^{\prime}$.

Proof. Suppose such a $\phi$ exists. Then by 3.4 there exists a matrix $P \in \mathrm{GL}(2, \mathbb{Z})$ with $P \geq 0$ such that $P^{-1} M P=M^{\prime}$. We know by 3.3 that $P=M_{1} M_{2} \cdots M_{k}$ where one of the following is true: (i) $k=1$ and $M_{1}=x$; (ii) $M_{i}$ is a shear matrix for $1 \leq i \leq k$; or (iii) $M_{i}$ is a shear for $1 \leq i \leq k-1$ and $M_{k}$ is either $y$ or $y^{\prime}$ where $x$ and $y$ are as in 3.2.

Case (i). Conjugating $M$ by $x$ corresponds to relabeling the rectangles; i.e. $R_{1}$ and $R_{2}$ and $R_{2}$ as $R_{1}$.

Case (ii). We know by Lemma 4.1 that conjugating $M$ by a shear matrix is "legal" as far as partitions go. Therefore, since $P^{-1} M_{k}^{-1} M_{k-1}^{-1} \cdots M_{1}^{-1}$, we need 
only show that $M_{j}^{-1} \cdots M_{1}^{-1} M M_{1} \cdots M_{j} \geq 0$ for $1 \leq j \leq k$. It is sufficient to show that $M_{1}^{-1} M M_{1} \geq 0$. So, suppose $P^{-1} M P \geq 0$ with $P$ as above. At this point note that multiplication on the right by a shear matrix does not change the dominant row of $M$ and hence, $M P$ and $M$ have the same dominant row. Therefore, since $M_{k}^{-1} \cdots M_{1}^{-1} M P \geq 0$, we know that $M_{1}^{-1} M P \geq 0$. Since $M_{1}^{-1}$ is the inverse of a shear matrix and $M P$ and $M$ have the same dominant row, $M_{1}^{-1} M \geq 0$ also. Hence $M_{1}^{-1} M M_{1} \geq 0$ which concludes case (ii).

Case (iii). If $M_{1}=y$ or $y^{\prime}$ then let $P^{\prime}=P x$. Now $P^{\prime}$ is a product of shear matrices and reduces to case (ii). We see, however, that $x P^{\prime-1} M P^{\prime} x$ is just $P^{-1} M P$ and hence by case (i) we have proven our proposition.

Now we give necessary and sufficient conditions on the Markov matrix for a hyperbolic automorphism in the two rectangle case.

Theorem 4.5. Let $\mathscr{A}$ be a hyperbolic automorphism of $\mathbb{T}^{2}$, and let $M$ be a nonnegative $2 \times 2$ integer matrix.

(i) If $\operatorname{Tr}(\mathscr{A})>0$, then there exists a Markov partition $\mathscr{P}$ for $\mathscr{A}$ with Markov matrix $M \Leftrightarrow$ there exists $\phi \in \mathrm{GL}(2, \mathbb{Z})$ such that $\phi^{-1} \mathscr{A} \phi=$ $M$.

(ii) If $\operatorname{Tr}(\mathscr{A})<0$, then there exists a Markov partition $\mathscr{P}$ for $\mathscr{A}$ with Markov matrix $M \Leftrightarrow$ there exists $\phi \in \mathrm{GL}(2, \mathbb{Z})$ such that $\phi^{-1} \mathscr{A} \phi=$ $-M$.

Proof. $(\Rightarrow)$ In both cases this is Corollary 2.4.

$(\Leftrightarrow)$ Suppose $\operatorname{Tr}(\mathscr{A})>0$, and suppose there exists $\phi \in \mathrm{GL}(2, \mathbb{Z})$ such that $\phi^{-1} \mathscr{A} \phi=M$. Let $\mathscr{P}^{\prime}$ be any Markov partition for $\mathscr{A}$ with two rectangles. $\mathscr{P}^{\prime}$ gives rise to a Markov matrix, call it $M^{\prime}$. By Corollary 2.4, there exists $\psi \in \mathrm{GL}(2, \mathbb{Z})$ such that $\psi^{-1} \mathscr{A} \psi=M^{\prime}$. Then $\phi^{-1} \psi M^{\prime} \psi^{-1} \phi=M \Rightarrow$ $\left(\psi^{-1} \phi\right)^{-1} M^{\prime}\left(\psi^{-1} \phi\right)=M \Rightarrow$ by Proposition 4.4 there exists a partition $\mathscr{P}$ for $\mathscr{A}$ with Markov matrix $M$. If $\operatorname{Tr}(\mathscr{A})<0$, let $\mathscr{P}^{\prime}$ be any Markov partition for $\mathscr{A}$ with two rectangles and Markov matrix $-M$, and Proposition 4.4 gives us the theorem as above.

\section{A CONJECTURE}

We know that the Markov matrix $M$ for any hyperbolic automorphism of $\mathbb{T}^{2}$ must be aperiodic (there is some integer $n \geq 1$ such that $M^{n}>0$ ) because the image of every rectangle is dense in $\mathbb{T}^{2}$. It is unknown whether or not this is enough for the converse of Corollary 2.3 to be true.

Conjecture 5.1. Let $\mathscr{A}$ be a hyperbolic automorphism of $\mathbb{T}^{2}(\operatorname{Tr}(\mathscr{A})>0)$ and let $M$ be an $n \times n$ integer matrix, $n>2$. Then there is a Markov partition $\mathscr{P}$ with $n$ rectangles for $\mathscr{A}$ with Markov matrix $M \Leftrightarrow M$ is aperiodic and 
$M$ is similar over $\mathbb{Z}$ to a matrix of the following form:

$$
\left[\begin{array}{cccc}
\mathscr{A} & 0 & 0 & 0 \\
* & A_{1} & 0 & 0 \\
* & * & \ddots & 0 \\
* & * & * & A_{k}
\end{array}\right]
$$

where at least one but at most two of the nonzero $A_{i}$ have eigenvalues the $p$ th roots of unity excluding 1 for some $p$, and the rest of the nonzero $A_{i}$ have eigenvalues $\{$ the $p$ th roots of unity for some $p$.

\section{REFERENCES}

[AW] R. L. Adler and B. Weiss, Similarity of automorphisms of the torus, Mem. Amer. Math. Soc. 98 (1970), 17-36.

[D] R. L. Devaney, An introduction to chaotic dynamical systems, Benjamin/Cummings, Menlo Park, CA, 1986.

[KR] K. H. Kim and F. W. Roush, Some results on decidability of shift equivalence, J. Combin. Inform. System Sci. (1979), 123-146.

[N] M. Newman, Integral matrices, Academic Press, New York, 1972, pp. 50-52.

[Sn] M. R. Snavely, Markov partitions for hyperbolic automorphisms of the two-dimensional torus, Ph.D. thesis, Northwestern Univ., 1990.

[St] M. W. Stafford, Markov partitions for the doubling map, Ph.D. thesis, Northwestern Univ., 1989.

Department of Mathematics, Northwestern University, Evanston, Illinois 60208 Current address: Department of Mathematics, Carthage College, Kenosha, Wisconsin 53140 E-mail address: snavely@rufus.math.nwu.edu 